\title{
Neurological involvement associated with COVID-19 disease: a study on psychosocial factors
}

\author{
Arianna Mariniello ${ }^{1} \cdot$ Silvia Schiavolin $^{1}$ (1) . Francesca Magnani ${ }^{1} \cdot$ Viviana Cristillo $^{2} \cdot$ Stefano Cotti Piccinelli $^{2}$. \\ Nicola Zoppi ${ }^{2}$. Giulio Bonzi ${ }^{2}$. Davide Sattin ${ }^{3}$. Fabiola Silvaggi ${ }^{1}$. Alberto Raggi ${ }^{1}$ - Martina Cacciatore ${ }^{1}$. \\ Erika Guastafierro ${ }^{1} \cdot$ Claudia Toppo $^{1} \cdot$ Stefano Gipponi $^{2} \cdot$ Ilenia Libri $^{2} \cdot$ Michela Bezzi $^{4} \cdot$ Matilde Leonardi $^{1}$. \\ Andrea Pilotto $^{2} \cdot$ Alessandro Padovani $^{2}$
}

Received: 21 December 2021 / Accepted: 17 January 2022 / Published online: 22 January 2022

(c) Fondazione Società Italiana di Neurologia 2022

\begin{abstract}
Background Several people affected by COVID-19 experienced neurological manifestations, altered sleep quality, mood disorders, and disability following hospitalization for a long time.

Objective To explore the impact of different neurological symptoms on sleep quality, mood, and disability in a consecutive series of patients previously hospitalized for COVID-19 disease.

Methods We evaluated 83 patients with COVID-19 around 3 months after hospital discharge. They were divided into 3 groups according to their neurological involvement (i.e., mild, unspecific, or no neurological involvement). Socio-demographic, clinical data, disability level, emotional distress, and sleep quality were collected and compared between the three groups.

Results We found that higher disability, depressive symptoms, and lower sleep quality in patients with mild neurological involvement compared to patients with unspecific and no neurological involvement. Differences between groups were also found for clinical variables related to COVID-19 severity.

Conclusion After 3 months from hospital discharge, patients with more severe COVID-19 and mild neurological involvement experienced more psychosocial alterations than patients with unspecific or no neurological involvement. Both COVID-19 and neurological manifestations' severity should be considered in the clinical settings to plain tailored interventions for patients recovering from COVID-19.
\end{abstract}

Keywords COVID-19 $\cdot$ Neurological manifestation $\cdot$ PSQI $\cdot$ HADS $\cdot$ WHODAS-12

Silvia Schiavolin

silvia.schiavolin@istituto-besta.it

1 Neurology, Public Health and Disability Unit, Fondazione IRCCS Istituto Neurologico Carlo Besta, Via Celoria 11, 20133 Milan, Italy

2 Neurology Unit, Department of Clinical and Experimental Sciences, University of Brescia, P.le Spedali Civili 1, 25123 Brescia, Italy

3 Hospital Health Direction, IRCCS Istituti Clinici Scientifici Maugeri, 20138 Milan, Italy

4 Respiratory Unit, ASST Spedali Civili Di Brescia, Brescia, Italy

\section{Introduction}

Coronavirus disease 19 (COVID-19) is a global pandemic caused by severe acute respiratory syndrome coronavirus 2 (SARS-CoV-2), with a severe impact on global public health since December 2019. In Italy, COVID-19 was experienced for the first time in the Lombardy region, on the 20 of February 2020.

The clinical spectrum of SARS-CoV-2 ranges from no manifesting symptoms to having symptoms like fever, cough, sore throat, weakness, fatigue, and muscle pain [1]. Furthermore, increasing evidence suggests that a substantial proportion of hospitalized COVID-19 patients exhibited neurological symptoms and disease affecting both central nervous system (CNS) and peripheral nervous system (PNS), such as headache, dizziness, impaired consciousness, acute cerebrovascular 
disease, epilepsy on one side, and hyposmia/anosmia, hypogeusia/ageusia, muscle pain, and Guillain-Barre syndrome on the other [2-6]. The most severe cases can be present with pneumonia, acute respiratory distress syndrome, and other complications, all of which could be lethal [7].

A recent study highlighted that COVID-19 patients reported a broad range of long-term neurological symptoms' manifestation after hospitalization. Specifically, age, premorbid conditions, and history of severe COVID-19 were found independent predictors of neurological manifestations [8].

Indeed, several people affected by COVID-19 can experience persistent symptoms. This phenomenon has been named "long COVID" [9], characterized by experiencing fatigue, muscle weakness, sleep difficulties, anxiety, and depression [10]. Most hospitalized patients with COVID19 diagnosis and with poor quality of sleep had a prolonged hospital length of stay and experienced a higher prevalence of depression than patients with good sleep quality [11]. Although a previous study analyzed the impact of COVID19 on the quality of sleep and sleep latency in the general population during the lockdown [12], few studies explored sleep quality in patients hospitalized with COVID-19 to our knowledge $[13,14]$. Poor sleep quality and more physical symptoms are, in turn, risk factors for severe anxiety [15]; moreover, patients with poor sleep also reported clinically significant psychiatric comorbidity and psychological distress following diagnosis, including protracted symptoms, past traumatic events, and negative view on the COVID-19 pandemic [16]. This evidence, taken together, highlights the importance to consider both sleep and mood dysregulation when caring for patients recovering from COVID-19.

Despite the growing evidence on long-term health-related consequences of COVID-19 after hospital discharge [10, 17, 18], the impact of COVID-19 when associated with neurological symptoms is still unclear. For this reason, it could be useful to consider the extent to which the presence of neurological symptoms affects sleep quality, anxiety, and depression in the medium-long term. Furthermore, disability as a long-term outcome measure of hospitalization due to COVID-19 is poorly evaluated.

The aim of this study was to investigate the relationship between neurological symptoms and psychosocial factors (i.e., sleep quality, anxiety, depression, and disability) in a cohort of COVID-19 survivors after hospital discharge.

\section{Methods}

\section{Participants}

A total of 105 patients discharged from the ASST Spedali Civili Hospital, Brescia, Italy between February 2020 and April 2020, were enrolled for follow-up examination up to 6 months. Only 83 patients (mean age \pm SD: 65.2: \pm 1.3 years; mean education \pm SD $11.1 \pm 4.6 ; 63$ male) completed the cognitive evaluation and patients' reported outcome measures (PROMs; see the following section for details) for the aim of this study. Patients with premorbid psychiatric and neurological conditions were excluded.

To explore differences in psychosocial variables between COVID-19 patients manifesting different neurological symptoms, we divided the whole sample into groups following the criteria described in the study by Fleisher and colleagues [19]. Specifically, the authors identified 4 different clusters, namely (i) no evidence of neurological involvement (no NIV), (ii) unspecific NIV (dizziness, headache, muscle pain, general weakness), (iii) mild NIV (impaired taste or smell, cranial nerve paresis), and (iv) severe NIV (acute cerebrovascular disease, seizure, myopathy, or neuropathy). This study was designed according to the ethical standards of the Declaration of Helsinki and received approval from the local ethics committee. Written informed consent was obtained from all patients prior to their participation in the study.

\section{Assessment}

Patients were evaluated with different scales addressing socio-demographic, clinical, and PROMs.

Specifically, during the hospitalization, we collected socio-demographic information (including age, gender, education levels, marital status, working situation, economic status before COVID-19, and the number of cohabitants) and the clinical variables consisting of (i) the clinical severity of COVID-19 evaluated through the BresciaCOVID Respiratory Severity Scale (BCRSS) [20], (ii) the symptoms' onset, (iii) the need of $\mathrm{O} 2$ therapy during the hospitalization, (iv) the total days of hospitalization, and (v) the premorbid patients' comorbidities along with their severity quantified through the Cumulative Illness Rating Scale (CIRS) [21].

After the hospital discharge (mean $\pm \mathrm{SD}=3.3 \pm 0.8$ months), we collected the patients' comorbidities and severity indices through the CIRS, the patients' global cognitive functioning by administering the Montreal Cognitive Assessment Battery (MOCA) [22], and the following PROMs:

1) The Hospital Anxiety and Depression Scale (HADS) [23] was administered to evaluate emotional distress; it is a 14-item self-rated instrument composed of two different subscales evaluating anxiety and depression, respectively. The score at each subscale ranges from 0 to 21 with scores higher than 10 indicatives of anxiety 
or depression [23]. The total score, consisting of the sum of the sub-score at each subscale, ranges from 0 to 42 .

2) The WHO Disability Assessment Schedule (WHODAS-12) [24] was used to quantify the disability level. It is a 12-item questionnaire providing a global score of disability considering the difficulties experienced by individuals due to health conditions in six areas of daily life (i.e., mobility, cognition, self-care, life activities, getting alone, and participation). Each item is rated on a 5-point Likert scale (from 1, i.e., no difficulty, to 5, i.e., completely difficult); the total score ranges from 0 to 100 with higher scores suggestive of severe disability levels (for total score computation, see 24).

3) The Pittsburgh Sleep Quality Index (PSQI) [25] was adopted to record the sleep quality referring to the past month. It is a 19-item self-reported questionnaire divided into 7 components, namely (i) subjective sleep quality, (ii) sleep latency, (iii) sleep duration, (iv) habitual sleep efficiency, (v) sleep disorder, (vi) use of sleeping drugs, and (vii) daytime dysfunction. The global PSQI score, ranging from 0 to 21, consists of the sum of the sub-scores of each component. Patients with a global PSQI score $\geq 5$ experience poor sleep quality, and they are identified as having sleep disturbances.

\section{Statistical analyses}

Descriptive statistics are reported for socio-demographic and clinical variables both for the whole sample and for the three groups of patients (no NIV, unspecific NIV, mild NIV) separately. Absolute numbers and percentages were used for categorical variables, while mean and standard deviation/median and interquartile range (IQR) were used for continuous ones.

The WHODAS-12, HADS (both total and sub-scores), and PSQI global score have been considered as dependent variables of interest separately (see Online Resource 1 for groups comparison on clinical variables).

The data distribution was explored by considering values of kurtosis and skewness, while the Shapiro-Wilk test was used to check for normality assumption. Since the normality assumption was violated for all the considered variables $(p<0.05)$, the three groups of patients were compared using the non-parametric Kruskal Wallis test; in case of a significant result, we performed Mann-Whitney test for multiple comparisons between groups. The significance level was set to $p<0.05$ for all the analyses.

SPSS 24.0 statistical software was used for all the statistical analyses.

\section{Results}

We divided the sample into three groups: no NIV $(n=24$ patients), unspecific NIV ( $n=37$ patients), and mild NIV ( $n=22$ patients). None of the patients enrolled in the present study exhibited the criteria for severe NIV.

Descriptive statistics for both the whole sample of patients and the three groups separately are summarized in Table 1.

Considering the whole sample, most of the patients had non-pathological PROMs scores.

The comparison between groups showed a significant difference in the WHODAS-12 total score $\left(\chi_{(2)}^{2}=17.19\right.$; $p<0.001)$, in the PSQI global score $\left(\chi_{(2)}^{2}=9.39\right.$; $p=0.009)$, in the HADS total score $\left(\chi_{(2)}^{2}=7.85\right.$; $p=0.02)$, and in the HADS Depression score $\left(\chi^{2}{ }_{(2)}=7.19\right.$; $p=0.02$ ).

Specifically, patients with mild NIV had higher WHODAS-12 score $($ median $=18.75)$ than both patients with unspecific NIV (median $=6.25 ; U=173 ; p=0.002$.) and no NIV (median $=4.16 ; U=61 ; p<0.001$ ) (see Fig. 1a).

The same pattern of results was found when performing post-hoc comparisons for PSQI global score. Indeed, patients with mild NIV had higher PSQI score $($ median $=8)$ than both patients with unspecific NIV (median $=6$; $U=239.5 ; p=0.023$ ) and no NIV (median $=5 ; U=121.5$; $p=0.004$ ) (see Fig. 1b).

When performing the post-hoc comparisons for the HADS total score, the only significant difference was found between mild NIV (median $=10.5$ ) and no NIV (median $=5 ; U=122.5 ; p=0.003$ ) (see Fig. 1c). This result was driven by the significant difference found between mild and no NIV for the HADS Depression score $(\mathrm{U}=133.5 ; p=0.006)$. In other words, patients with mild NIV showed higher HADS depression score (median $=5$ ) than patients with no NIV (median =2) (see Fig. 1c).

No significant difference between groups were found in the HADS anxiety score $\left(\chi_{(2)}^{2}=4.16 ; p=0.12\right)$.

The three groups of patients also differed for some clinical variables as reported in the Online Research 1. Specifically, the distribution of cases undergoing to $\mathrm{O} 2$ therapy compared to who did not undergo to $\mathrm{O} 2$ therapy was greater in the mild NIV group than in the other groups. Similarly, mild NIV patients showed higher clinical severity of COVID-19 evaluated through the BCRSS than both no NIV $(p=0.003)$ and unspecific NIV $(p<0.001)$. Furthermore, these patients had a longer hospital stay than both no NIV $(p=0.001)$ and unspecific NIV $(p=0.001)$. Finally, mild NIV patients showed higher comorbidity index at admission than no NIV patients $(p=0.02)$ as well as both higher comorbidity $(p=0.04)$ and severity $(p=0.007)$ indices after 6 months compared to no NIV patients. 
Table 1 Socio-demographic, clinical and PROMs data in both the whole sample and the three groups of patients (No NIV, unspecific NIV, mild NIV); number and percentage for categorical variables; mean \pm standard deviation/median; and interquartile range for continuous variables

\begin{tabular}{|c|c|c|c|c|}
\hline \multirow[b]{2}{*}{ Variables } & \multirow[b]{2}{*}{ Tot } & \multicolumn{3}{|c|}{ Neurological involvement (NIV) } \\
\hline & & No & Mild & Unspecific \\
\hline \multicolumn{5}{|l|}{ Sociodemographic } \\
\hline Gender (male) & $75.9 \%(63)$ & $83.3 \%(20)$ & $59.1 \%(13)$ & $81.1 \%(30)$ \\
\hline Age (years) & $65.2 \pm 1.3$ & $63.2 \pm 11.1$ & $70.4 \pm 10.11$ & $63.3 \pm 13.1$ \\
\hline Education (years) & $11.1 \pm 4.6$ & $12.7 \pm 5$ & $9.3 \pm 4.5$ & $11.1 \pm 4$ \\
\hline \multicolumn{5}{|l|}{ Marital status } \\
\hline Single & $8.4 \%(7)$ & $16.7 \%(4)$ & $4.5 \%(1)$ & $5.4 \%(2)$ \\
\hline Married/Cohabitant & $81.9 \%(68)$ & $70.8 \%(17)$ & $72.7 \%(16)$ & $94.5 \%(35)$ \\
\hline Divorced/separated & $4.8 \%(4)$ & $4.2 \%(1)$ & $13.6 \%(3)$ & $0 \%(0)$ \\
\hline Widower & $4.8 \%(4)$ & $8.3 \%(2)$ & $9.1 \%(2)$ & $0 \%(0)$ \\
\hline \multicolumn{5}{|l|}{ Working situation } \\
\hline Employed & $34.9 \%(29)$ & $41.7 \%(10)$ & $27.3 \%(6)$ & $35.1 \%(13)$ \\
\hline Unemployed & $6 \%(5)$ & $0 \%(0)$ & $9.1 \%(2)$ & $8.1 \%(3)$ \\
\hline Retired & $59 \%(49)$ & $58.3 \%(14)$ & $63.6 \%(14)$ & $56.8 \%(21)$ \\
\hline \multicolumn{5}{|l|}{ Economic status (before COVID-19) } \\
\hline Well-off & $2.4 \%(2)$ & $0 \%(0)$ & $0 \%(0)$ & $5.4 \%(2)$ \\
\hline Medium & $61.4 \%(51)$ & $70.8 \%(17)$ & $72.7 \%(16)$ & $48.6 \%(18)$ \\
\hline Sufficient & $34.9 \%(29)$ & $29.2 \%(7)$ & $22.7 \%(5)$ & $45.9 \%(17)$ \\
\hline Insufficient & $1.2 \%(1)$ & $0 \%(0)$ & $4.5 \%(1)$ & $0 \%(0)$ \\
\hline \multicolumn{5}{|l|}{ Cohabitants } \\
\hline None & $12 \%(10)$ & $16.6 \%(4)$ & $22.7 \%(5)$ & $2.7 \%(1)$ \\
\hline One & $45.8 \%(38)$ & $33.3 \%(8)$ & $36.3 \%(8)$ & $59.4 \%(22)$ \\
\hline Two or more & $42.2 \%(35)$ & $50 \%(12)$ & $40.9 \%(9)$ & $37.8 \%(14)$ \\
\hline \multicolumn{5}{|l|}{ Clinical } \\
\hline Hospital stay (days) & $11.5 \pm 8.6$ & $9.2 \pm 5.9$ & $17.6 \pm 12.3$ & $9.3 \pm 5.2$ \\
\hline O2 therapy & $79.5 \%(66)$ & $75 \%(18)$ & $100 \%(22)$ & $70.2 \%(26)$ \\
\hline CIRS severity index admission (median and IQR) & $1.3 \pm 0.4$ & $1.3 \pm 0.3$ & $1.5 \pm 0.4$ & $1.3 \pm 0.4$ \\
\hline CIRS severity index follow-up (median and IQR) & $1.5 \pm 0.3$ & $1.3 \pm 0.3$ & $1.6 \pm 0.3$ & $1.5 \pm 0.3$ \\
\hline CIRS comorbidity index admission (median and IQR) & $2 \pm 2$ & $2 \pm 2$ & $3 \pm 3$ & $2 \pm 2$ \\
\hline CIRS comorbidity index follow-up (median and IQR) & $2 \pm 2$ & $2 \pm 3$ & $3 \pm 2$ & $2 \pm 2$ \\
\hline BCRSS & $.76 \pm .75$ & $.63 \pm .64$ & $1.30 \pm .76$ & $.50 \pm .65$ \\
\hline MOCA (total score) & $23.52 \pm 3.55$ & $25.54 \pm 2.53$ & $20.34 \pm 3.54$ & $24.19 \pm 2.73$ \\
\hline \multicolumn{5}{|l|}{ PROMs } \\
\hline WHODAS-12 & $6.2 \pm 14.6$ & $4.1 \pm 8.3$ & $18.7 \pm 21.9$ & $6.2 \pm 10.4$ \\
\hline HADS (total score) & $6 \pm 9$ & $5 \pm 6$ & $10.5 \pm 9$ & $6 \pm 9$ \\
\hline HADS (depression score) & $2.5 \pm 5$ & $2 \pm 4$ & $5 \pm 5$ & $2 \pm 6$ \\
\hline HADS (anxiety score) & $3.5 \pm 4$ & $3 \pm 4$ & $4 \pm 5$ & $3.5 \pm 5$ \\
\hline PSQI (total score) & $6 \pm 4$ & $5 \pm 3$ & $8 \pm 5$ & $6 \pm 3$ \\
\hline
\end{tabular}

\section{Discussion}

This exploratory study investigated the relationship between neurological symptoms and psychosocial factors in adult patients after COVID-19. Despite increasing evidence are showing the long-term consequences of COVID-19 [26], none had explored whether having different neurological manifestations associated with COVID-19 can lead to different long-term consequences, to our knowledge. Specifically, we aimed at exploring mood dysregulation, sleep disturbances, and perceived disability level after recovering from COVID-19 in a sample of patients who presented different neurological manifestations.

Overall, most patients did not show impoverishment of quality of sleep and pathological levels of disability, anxiety, or depression after hospital discharge.

Considering mood dysregulation, recent evidence showed an improvement of depressive symptoms and 
a)
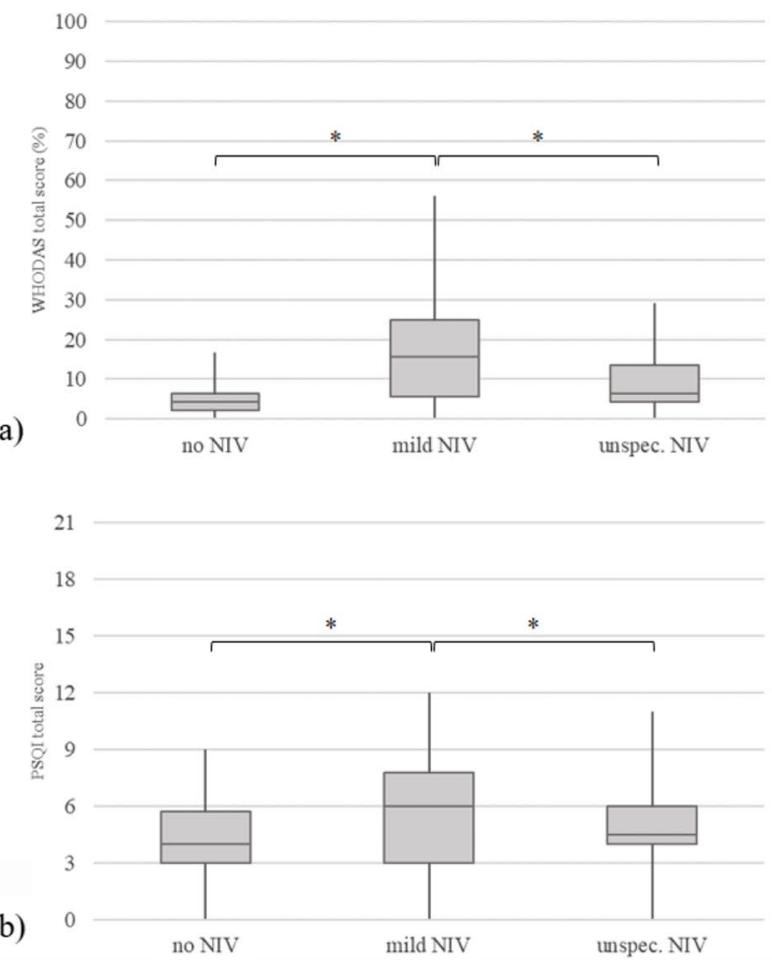

Fig. 1 The figure shows the box-plots of the PROMs for each group of patients. a represents the box-plots of the WHODAS-12 total score in percentage. b represents the box-plots of the Pittsburgh Sleep Quality Index (PSQI) total score. c represents the box-plots of the

anxiety in COVID-19 patients over time [27, 28]; thus, our findings on mood dysregulation, despite being assessed with different instruments, could be explained by the fact that the patients enrolled in the present study have been assessed for depression and anxiety a quite long time after hospital discharge. However, other studies went in a different direction showing higher rates of anxiety and depression at 4-6 months from recovery for COVID-19. Due to the heterogeneous results, future studies should monitor the mood dysregulation symptoms over time to better define whether increase or decrease of anxiety and depression can be a direct long-term consequence of COVID-19. As for sleep quality, a recent study exploring the longterm consequences of COVID-19 in a wider time window (6 months from hospital discharge) [10] described 1733 adult patients diagnosed with COVID-19 who manifested sleep difficulties ( $26 \%$ of the entire sample) when assessed through self-reported questionnaires on symptoms' prevalence [10]. Another recent study reported a very similar prevalence for sleep disturbances (19.23\%) after around 4 months from COVID-19 diagnosis in patients who did not require hospitalization [17]. With the present study, we confirmed this prevalence for sleep disorders by adopting a tailored instrument able to detect the quality of sleep of neurological patients after hospital discharge.

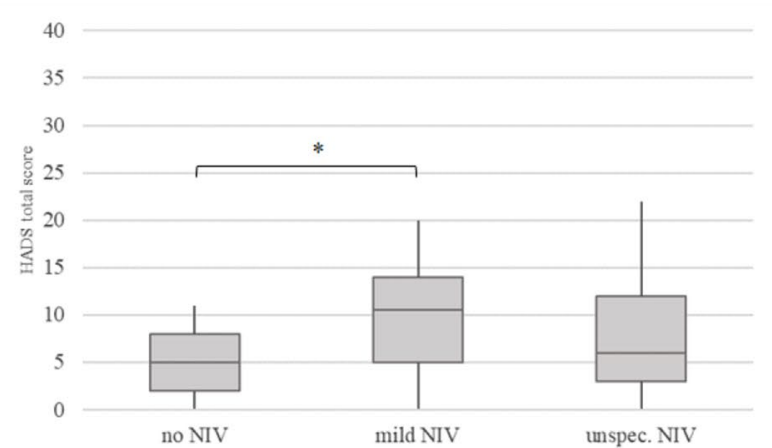

c)

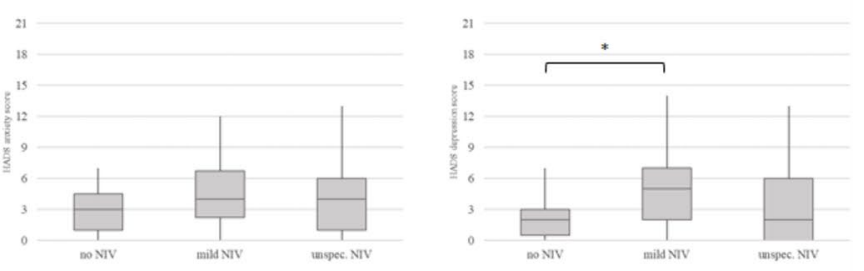

Hospital Anxiety and Depression Scale (HADS) total score (upper part), and both the HADS anxiety and depression score (lower part). Stars represent a significant difference $(p<0.05)$

When comparing patients belonging to different neurological clusters, we found that patients having mild NIV experienced a higher disability level and lower quality of sleep than both patients with unspecific and no NIV. Furthermore, mild NIV patients manifested higher levels of depression than no NIV ones. These results are quite surprising as dizziness, headache, muscle pain, and general weakness, characterizing unspecific NIV patients, could hypothetically affect the considered PROMs to a greater extent than experiencing loss of taste or smell (typical of mild NIV patients) or not experiencing the above-mentioned neurological manifestations at all. For instance, people who suffered from headache or dizziness manifested lower sleep quality and a higher rate of depression and anxiety than healthy individuals [29, 30]. In other words, the neurological symptoms' characterization of our patients seems not to explain why they differed in the considered PROMs. However, a possible explanation arises from the groups' comparison on the collected clinical variables (Online Research 1). Indeed, we found differences in several clinical variables indicating greater severity of COVID-19 in mild NIV patients rather than the other groups, as proved by higher scores at the BCRSS, longer hospital stays, and the use of oxygen therapy for the totality of the mild NIV patients. A 
recent study highlighted a similar rate of sleep disorders at 6-month follow-up for both patients requiring and not requiring oxygen therapy [10]; the authors also reported increasing prevalence of mood dysregulation as a function of COVID-19 severity: The authors concluded that illness severity had a key role in determining persistent psychological symptoms [10]. Our results went in this same direction and added evidence for the impact of illness severity also on the perceived disability level in time.

Usually, more severe neurological manifestations occur in patients with higher COVID-19 severity [31, 32], thus producing confounding results on the role of neurological manifestations on COVID-19 long-term consequences. We explored the influence of these two factors independently, and we supposed that higher levels of perceived disability, sleep, and mood dysregulation could be related to the COVID-19 severity rather than to the specific neurological manifestation. As a matter of fact, previous studies have already demonstrated that those who had a history of severe COVID-19 disease also showed a higher level of depressive and anxiety symptoms, and protracted sleep difficulties [10], and this seems true in patients displaying neurological symptoms. Finally, since we found mild NIV patients manifesting a higher number of comorbidities during the hospitalization and higher comorbidities' severity in the follow-up compared to patients with no evidence of neurological involvement, considering both the clinical course and the general medical conditions of patients with COVID-19 should be recommended when planning interventions aimed at reducing disability, mood, and sleep dysregulation in the long term.

\section{Limitations}

Some limitations should be discussed. Indeed, we did not collect PROMs at the baseline so that we were not able to assess any difference between the pre- and post-hospitalization periods, therefore we did not know whether mood and sleep disturbances, as well as high levels of perceived disability, were already present before COVID19 infection in some patients. Furthermore, the small sample size and the heterogeneity between groups, along with the cross-sectional design of the study, greatly undermine the causal inference. All these limitations are mainly due to the clinical setting where patients have been enrolled during the emergency. Future studies should explore with different experimental designs and statistical models what weight COVID-19 and neurological manifestations severity have in predicting the perceived disability level, mood, and sleep dysregulation in the long term. This is needed to help clinicians in planning interventions for patients recovering from COVID-19.

\section{Conclusions}

When comparing patients belonging to different neurological clusters, we found that patients having mild neurological symptoms experienced a higher disability level and lower quality of sleep than both patients with unspecific and not having neurological involvement after more than three months post-discharge. On one hand, this might indicate a burden of mild neurological manifestations on disability, as perceived by patients. On the other, neurological symptoms might be triggered by a higher stress level and burden of patients. This last concept fit with the discordance we found between the common subjective symptoms and the rare objective neurological alterations we found in the same cohort of patients.

The present findings claim indeed for a strong relationship between neurological manifestations, psychosocial burden, and perceived disability. Furthermore, they pointed out once again both premorbid conditions and severity of COVID-19 disease as important predictors of long COVID manifestations. Identifying the physiological and psychological mechanisms underpinning neurological manifestations and explaining the self-reported disability is an important step for identifying specific target population for possible nonpharmacological interventions.

Supplementary Information The online version contains supplementary material available at https://doi.org/10.1007/s10072-022-05907-1.

Acknowledgements The authors thank all the participants for their participation.

Data availability The datasets generated and analyzed during the current study are available from the corresponding author on reasonable request.

\section{Declarations}

Ethical approval Approval was obtained by the local ethics committee of ASST "Spedali Civili di Brescia" Hospital.

Consent to participate Informed consent was obtained from all individual participants included in the study.

Conflict of interest Andrea Pilotto served on the advisory board of Zcube (technology division of Zambon pharmaceuticals); he received honoraria from Z-cube s.r.l., Biomarin, Zambon, Abbvie, Nutricia and Chiesi pharmaceuticals. He received research support from Vitaflo Germany and Zambon Italy. Alessandro Padovani is consultant and served on the scientific advisory board of GE Healthcare, Eli-Lilly and Actelion Ltd. Pharmaceuticals and received speaker honoraria from Nutricia, PIAM, Langstone Technology, GE Healthcare, Lilly, UCB Pharma and Chiesi Pharmaceuticals. He is funded by grant of the Ministry of University (MURST).

The other authors declare no competing interests. 


\section{References}

1. Wang D, Hu B, Hu C et al (2020) Clinical characteristics of 138 hospitalized patients with 2019 novel coronavirus-infected pneumonia in Wuhan, China. JAMA 323(11):1061-1069. https://doi. org/10.1001/jama.2020.1585

2. Werner C, Scullen T, Mathkour M et al (2020) Neurological impact of coronavirus disease of 2019: practical considerations for the neuroscience community. World Neurosurg 139:344-354. https://doi.org/10.1016/j.wneu.2020.04.222

3. Mao L, Jin H, Wang M et al (2020) Neurologic manifestations of hospitalized patients with coronavirus disease 2019 in Wuhan, China. JAMA Neurol 77(6):683-690. https://doi.org/10.1001/ jamaneurol.2020.1127

4. Li Y-C, Bai W-Z, Hashikawa T (2020) The neuroinvasive potential of SARS-CoV2 may play a role in the respiratory failure of COVID-19 patients. J Med Virol 92(6):552-555. https://doi.org/ 10.1002/jmv. 25728

5. Helms J, Kremer S, Merdji H et al (2020) Neurologic Features in severe SARS-CoV-2 infection. N Engl J Med 382(23):2268-2270. https://doi.org/10.1056/NEJMc2008597

6. Niazkar HR, Zibaee B, Nasimi A, Bahri N (2020) The neurological manifestations of COVID-19: a review article. Neurol Sci 41(7):1667-1671. https://doi.org/10.1007/s10072-020-04486-3

7. Gibson PG, Qin L, Puah SH (2020) COVID-19 acute respiratory distress syndrome (ARDS): clinical features and differences from typical pre-COVID-19 ARDS. Med J Aust 213(2):54-56. https:// doi.org/10.5694/mja2.50674

8. Pilotto A, Cristillo V, Piccinelli SC et al (2021) Long-term neurological manifestations of COVID-19: prevalence and predictive factors. Neurol Sci 398(10302):725. https://doi.org/10.1016/ S0140-6736(21)01900-0

9. Lancet T (2021) Understanding long COVID: a modern medical challenge. Lancet (London, England) 398(10302):725. https://doi. org/10.1016/S0140-6736(21)01900-0

10. Huang C, Huang L, Wang Y et al (2021) 6-month consequences of COVID-19 in patients discharged from hospital: a cohort study. Lancet (London, England) 397(10270):220-232. https://doi.org/ $10.1016 /$ S0140-6736(20)32656-8

11. Akınc1 T, Melek BH (2021) Relationship between sleep quality and the psychological status of patients hospitalised with COVID19. Sleep Med 80:167-170. https://doi.org/10.1016/j.sleep.2021. 01.034

12. Marelli S, Castelnuovo A, Somma A et al (2021) Impact of COVID-19 lockdown on sleep quality in university students and administration staff. J Neurol 268(1):8-15. https://doi.org/10. 1007/s00415-020-10056-6

13. Liu K, Chen Y, Wu D, Lin R, Wang Z, Pan L (2020) Effects of progressive muscle relaxation on anxiety and sleep quality in patients with COVID-19. Complement Ther Clin Pract 39:101132. https://doi.org/10.1016/j.ctcp.2020.101132

14. Vitale JA, Perazzo P, Silingardi M, Biffi M, Banfi G, Negrini $F$ (2020) Is disruption of sleep quality a consequence of severe Covid-19 infection? A case-series examination. Chronobiol Int 37(7):1110-1114. https://doi.org/10.1080/07420528.2020.17752 41

15. Dai LL, Wang X, Jiang TC et al (2020) Anxiety and depressive symptoms among COVID-19 patients in Jianghan Fangcang Shelter Hospital in Wuhan, China. PLoS One 5(8):e0238416. https:// doi.org/10.1371/journal.pone.0238416

16. Poyraz BÇ, Poyraz CA, Olgun Y et al (2021) Psychiatric morbidity and protracted symptoms after COVID-19. Psychiatry Res. https://doi.org/10.1016/j.psychres.2020.113604

17. Henneghan AM, Lewis KA, Gill E et al (2021) Describing cognitive function and psychosocial outcomes of COVID-19 survivors: a cross-sectional analysis. J Am Assoc Nurse Pract. https://doi. org/10.1097/JXX.0000000000000647

18. Zhang X, Wang F, Shen Y et al (2021) Symptoms and health outcomes among survivors of covid-19 infection 1 year after discharge from hospitals in Wuhan, China. JAMA Netw open 4(9):e2127403. https://doi.org/10.1001/jamanetworkopen.2021.27403

19. Fleischer M, Köhrmann M, Dolff S et al (2021) Observational cohort study of neurological involvement among patients with SARS-CoV-2 infection. Ther Adv Neurol Disord 14:1756286421993701. https://doi.org/10.1177/1756286421 993701

20. Piva S, Filippini M, Turla F et al (2020) Clinical presentation and initial management critically ill patients with severe acute respiratory syndrome coronavirus 2 (SARS-CoV-2) infection in Brescia, Italy. J Crit Care 58:29-33. https://doi.org/10.1016/j.jcrc.2020.04. 004

21. Linn BS, Linn MW, Gurel LEE (1968) Cumulative illness rating scale. J Am Geriatr Soc 16(5):622-626

22. Santangelo G, Siciliano M, Pedone R et al (2015) Normative data for the Montreal cognitive assessment in an Italian population sample. Neurol Sci Off J Ital Neurol Soc Ital Soc Clin Neurophysiol 36(4):585-591. https://doi.org/10.1007/s10072-014-1995-y

23. Costantini M, Musso M, Viterbori P et al (1999) Detecting psychological distress in cancer patients: validity of the Italian version of the hospital anxiety and depression scale. Support Care Cancer Off J Multinatl Assoc Support Care Cancer 7(3):121-127. https://doi.org/10.1007/s005200050241

24. Andrews G, Kemp A, Sunderland M, Von Korff M, Ustun TB (2009) Normative data for the 12 item who disability assessment schedule 2.0. PLoS One 4(12):e8343. https://doi.org/10.1371/ journal.pone.0008343

25. Curcio G, Tempesta D, Scarlata S et al (2013) Validity of the Italian version of the Pittsburgh Sleep Quality Index (PSQI). Neurol Sci Off J Ital Neurol Soc Ital Soc Clin Neurophysiol 34(4):511519. https://doi.org/10.1007/s10072-012-1085-y

26. Del Rio C, Collins LF, Malani P (2020) Long-term health consequences of COVID-19. JAMA 324(17):1723-1724. https://doi. org/10.1001/jama.2020.19719

27. Mazza MG, De Lorenzo R, Conte C et al (2020) Anxiety and depression in COVID-19 survivors: role of inflammatory and clinical predictors. Brain Behav Immun 89:594-600. https://doi. org/10.1016/j.bbi.2020.07.037

28. Matalon N, Dorman-Ilan S, Hasson-Ohayon I et al (2021) Trajectories of post-traumatic stress symptoms, anxiety, and depression in hospitalized COVID-19 patients: a one-month follow-up. J Psychosom Res 143:110399. https://doi.org/10.1016/j.jpsychores. 2021.110399

29. Boardman HF, Thomas E, Millson DS, Croft PR (2005) Psychological, sleep, lifestyle, and comorbid associations with headache. Headache 45(6):657-669. https://doi.org/10.1111/j.1526-4610. 2005.05133.x

30. Sugaya N, Arai M, Goto F (2017) The effect of sleep disturbance in patients with chronic dizziness. Acta Otolaryngol 137(1):4752. https://doi.org/10.1080/00016489.2016.1213418

31. Leonardi M, Padovani A, McArthur JC (2020) Neurological manifestations associated with COVID-19: a review and a call for action. J Neurol 267(6):1573-1576. https://doi.org/10.1007/ s00415-020-09896-Z

32. Kremer S, Lersy F, de Sèze J et al (2020) Brain MRI findings in severe COVID-19: a retrospective observational study. Radiology 297(2):E242-E251. https://doi.org/10.1148/radiol.2020202222

Publisher's note Springer Nature remains neutral with regard to jurisdictional claims in published maps and institutional affiliations. 